\title{
Intercellular cancer collisions generate an ejected crystal comet tail effect with fractal interface embryoid body reassembly transformation
}

This article was published in the following Dove Press journal:

Cancer Management and Research

7 May 2011

Number of times this article has been viewed

Jairo A Díaz

Mauricio F Murillo

Alvaro Barrero

Department of Pathology, Hospital Departmental Villavicencio,

Hospital Departmental Granada,

Medicine School, University

Cooperative of Colombia,

Villavicencio, Meta, Colombia
Correspondence: Jairo A Díaz

Department of Pathology, University Cooperative of Colombia Medicine

School, Km 2 Via Acacias, Colombia

Tel +II 5786628905

Fax +II 5786628905

Email jaditod@hotmail.com
Abstract: We have documented self-assembled geometric triangular chiral crystal complexes (GTCHC) and a framework of collagen vascular invariant geometric attractors in cancer tissues. This article shows how this system evolves in time. These structures are incorporated together and evolve in different ways. When the geometric core is stable, and the tissue architecture collapses, fragmented components emerge, which reveal a hidden interior identifying how each molecule is reassembled into the original mold, using one common connection, ie, a fractal self-similarity that guided the system from the beginning. GTCHC complexes generate ejected crystal comet tail effects and produce strange helicity states that arise in the form of spin domain interactions. As the crystal growth vibration stage progresses, biofractal echo images converge in a master-built construction of embryoid bodies with enolase-selective immunopositivity in relation to clusters of triangular chiral cell organization. In our electro-optic collision model, we were able to predict and replicate all the characteristics of this complex geometry that connects a physical phenomenon with the signal patterns that generate biologic chaos. Intrinsically, fractal geometry makes spatial correction errors embrace the chaotic system in a way that permits new structures to emerge, and as a result, an ordered self-assembly of embryoid bodies with neural differentiation at the final stage of cancer development is a predictable process. We hope that further investigation of these structures will lead not only to a new way of thinking about physics and biology, but also to a rewarding area in cancer research.

Keywords: embryoid bodies, cancer, electro-optic collision model

\section{Introduction}

In 1963, George Rose from the Biology Department, The University of Texas, identified for the first time unusually large extracellular crystals and particles produced by embryonic chick cells in special tissue culture environments in various forms; helical, tubular, ribbon-like, triangular, hexagonal, rhomboidal, and filamentous. ${ }^{1}$ The origin of these forms were detailed biochemically but their usefulness in the culture environments in which they were found remained obscure, although temptingly speculative, at that time. ${ }^{2}$

A self-assembly of geometric triangular chiral hexagon crystal-like complex organizations (GTCHC) has also been described in pathological human tissues. ${ }^{3}$ The authors have found this architectural geometric expression at both macroscopic and microscopic levels, mainly in cancer processes. Molecular crystals, represented by triangular chiral hexagons derived from a collision-attraction event against collagen type I fibrils, emerge at microscopic and macroscopic scales, presenting a lateral assembly at each side of hypertrophic helicoid fibers, which represent energy 
flow in cooperative hierarchically chiral electromagnetic interactions in cancer tissues and arise as geometry of the equilibrium in perturbed biological systems. Stimulated collagen type I fibers at the moment of collision result in hypertrophy of helicoid patterns, and their molecular crystal components are fractioned, being ejected to each side of the bundle fibril axis, generating polarization and straight lines of chirality in the GTCHC complex. Polarization, chirality, helicoid patterns, and geometric expression can be in intimate relation with cancer collision events, and the electromagnetic field that it generates. The laws of physics are amazingly universal because they serve as common denominators for dissimilar systems.

Additionally, the magnetic field in cancer tissues, and how it generates a functional geometric attractor complex in their entangled environment, has been documented. ${ }^{4}$ When the crystal images documented by George Rose were compared with our GTCHC complexes, these were identical, principally in relation to the presence of triangular chiral mirror images and fractals in the tissue environment. Human embryo and neoplastic tissue cultures similarly are being evaluated for their megaparticulate productivity. Recent experiments have displayed their capacity to give rise to similar forms. It is clear that these complexes are not the final process of inconclusive geometry or a frustrated system organization.

Using a computational model, Nagpal et al demonstrated that the regularity of the tissue, such as the percentage of hexagons and the overall cell shape distribution, can act as an indicator for inferring the properties of the cell division mechanism in epithelial tissues of growing organisms, from fruit flies to humans. ${ }^{5}$ Furthermore, the ability to cope with often unpredictable variations (referred to as "robustness") is critical for normal development. Fast growth, such as in cancer, entails numerous cell divisions, which must be balanced with the proper regulation of overall tissue space and organ architecture. Yet, how does the organism cope with space in this situation? Examination of the natural history of tissue assembled in the final stages of cancer development gives us a clue about the assembly process. In this study, we have illustrated this order assemblage only in the final products that were totally visible in order to gauge the processes behind those products by ensemble averages of many molecules, as well as to understand how extreme chaos at one pole of the system can generate extreme order at the opposite pole.

With increased observation in cancer tissues, we could comprehend their nature. Hexagonal geometry represents the maximal shape interpretation of equilibrium and is the highest expression of chirality in all dynamic biologic systems. Solving and understanding triangular chiral hexagons, such as crystal complexes (GTCHC), and their sequential development, represent a powerful tool to comprehend the direct mechanism by which the structural blocks organize in this hidden order.

Three very fundamental characteristics are integrated, ie, self-assembly, crystal complexes, and chirality. It is surprising that in the middle of structural chaos, disorganization, genetic mutations, and autonomous cell proliferation activity can emerge, as occurs in cancer. In biology, self-assembly describes processes in which a disordered system with preexisting components forms an organized structure or pattern as a consequence of specific local interactions among the components themselves; without external direction, the self-assembled structure must have a higher order. A direct consequence is the general tendency of self-assembled structures to be relatively free of defects, and this is referred to the minimum number of units needed to make an order. Cairns-Smith formulated his theory of crystalline ancestry, ${ }^{6}$ which accounts for the main mechanism by which life can form from inorganic precursors. Additionally, recent research at Yale provided a glimpse of the ancient mechanism that helped diversify our genomes, and has illuminated a relationship between gene processing in humans and the most primitive organisms by creating the first crystal structure of a crucial self-splicing region of RNA. ${ }^{7}$ In another way, many biologically active molecules are chiral, including naturally occurring amino acids and sugars. The obvious question to ask here is what are these characteristics related to speciation, specialization, complexity, and ordered building blocks doing in the microenvironment of cancer? Such geometric order found in a chaotic system tells us indirectly that we are faced with an organization. What happens to the crystal particles released in this collision event? How do such systems evolve over time?

\section{Materials and methods}

This study was approved by the ethical subcommittee of the Faculty of Medicine, University Cooperative of Colombia and followed the guidelines of the Minister of Health No 8430 of 1993 in accordance with the Declaration of Helsinki. All patients had signed an informed consent form for use of their biologic materials for diagnostic and research purposes.

We collected and re-examined all our materials, and identified triangular chiral crystals and geometric attractors, corresponding to more than $1077 \mathrm{microscopic} / \mathrm{macroscopic}$ specimens of different kinds of cancer tissues collected 
in the past 5 years. ${ }^{5}$ To our surprise, we found that these earlier images were only the tip of the iceberg, indicating the existence of a new complete invariant structural diffraction stripe line-related crystal geometrical assemblage in cancer tissues.

Based on the premise of observing cancer at the final stage, because tissue assembled as part of the natural tissue gives us a clue regarding the assembly process used, 90 fresh specimens of different tumors, principally of a silent malignant retroperitoneal type, including renal, ovary dysgerminomas, malignant fibrohistiocytomas, malignant hemangiopericytomas, and leiomyosarcomas, were added to the old material. All the specimens were of voluminous mass, varying from $15 \mathrm{~cm}$ to $20 \mathrm{~cm}$ in diameter, and were not medically manipulated because most of these tumors were from patients in rural areas.

We collected tumors directly from the surgical center before they were placed in formaldehyde. The reason for this is that we had observed that formaldehyde would destroy and eliminate an important part of the geometric crystal complexes that we were aiming to identify. It is at the external boundaries of the tumor where the largest molecular collisions are carried out. This interface zone corresponds to serosal surfaces in which we identified numerous GTCHC and geometric attractors. We washed each surgical specimen with water. The best way to study these structures is in their wet natural environment; if they are exposed to air, they will collapse. We collected the resulting products in glass containers, the material was filtered, and the mucous gelatinous component was separated out. Subsequently, the material was examined in a dark room with white light. We observed that under light reflection, some gelatinous components of the mucous material reflected light and were bright in the dark environment. These components were put onto a glass slide and stained with Papanicolaou stain. Tissue samples, in which the geometric complex assemblage was observed, were stained with hematoxylin and eosin, and with neuronspecific enolase immunostain. These structures have not been identified in normal tissues.

\section{Neuron-specific enolase immunostain}

In our previous research, we documented how vascular immunostain factor VIII-related antigen could increase and facilitate the identification of geometric collagen vascular frameworks in malignant tissues. Polar triangular vascular contraction lumina showed high immunoreactivity, whereas mirror triangular vascular dilatation lumina at the opposite pole showed total absence or low immunopositivity with respect to the reference antibody. Our interest was to identify the nature of this negative reactivity of the polar mirror triangular vascular geometry. We used enolase 2 (NSE, gamma neuronal), also known as ENO2. This isoenzyme is a homodimer found in mature neurons and cells of neuronal origin. ${ }^{8}$ Sixty formalin-fixed and paraffin-embedded tissue sections with the most representative hot spot triangular chiral mirror geometry complex areas were analyzed using neuron-specific enolase antibody marker. We performed immunohistochemistry using the standard protocol method with paraffin sections. ${ }^{9}$ The scoring was done as follows: $\mathrm{Ni}$ (no immunostain); low (10\% or less immunopositivity); or high $(>10 \%$ immunoreactive cells).

\section{Electro-optical model}

It is difficult to carry out an appropriate methodological observation for geometry-related electromagnetic assemblage processes when studying biological systems. However, one can obtain indirect information from models from other dynamic systems. We attempted to determine whether one could reproduce and predict patterns similar to those detected in association with the GTCH complexes in cancer tissues through electromagnetic sequential collisions.

To produce this effect, we used the standard methodology described earlier. The electro-optical model consists of an electronic flash device attached to a Sony camera (DSC-S600; Tokyo, Japan). Strong discharge pulses of light were sent over electric conduction lines $(150 \mathrm{~V})$ in a helical pattern. The time intervals were $3-4$ minutes, and the light discharges were sent in cycles of 60 minutes from a distance of 3-4 m, in an atmospheric environment and at a low temperature of $4^{\circ} \mathrm{C}$. There were 1 hour sessions over 9 days. To increase the generation and frequency of complete hexagonal patterns and their dynamic assembly, we changed the time period in which the experiment was performed. Previously, it was in complete darkness, but was subsequently changed to dawn between 5.00 AM and 5.30 AM when the first light rays appear on the evaluated magnetic system.

\section{Statistical analysis}

Interrelations between GTCH complexes and the crystal comet effect tail (CCET) assemblage in cancer tissues were determined. The relationship between CCET assemblage and embryoid body transformation was estimated, as well as neuron-specific enolase antibody immunostain positivity index in correlation with embryoid transformation areas. Chi-squares for the proportions were estimated using 
EPI-INFO software (v 6.04; Centers for Disease Control and Prevention, Atlanta, GA).

\section{Results}

In the cumulative visual information analysis of more than 1167 macroscopic and microscopic cancer tissue specimens, we found triangular chiral crystal images and a framework of collagen geometric attractors. We also observed that these structures are incorporated together and form part of an integrated geometric complex; they evolve in different ways where the geometric core is stable, whereas in others, it grows in proportion to the system, challenging our ability to understand all the possible component interactions. Our findings represent a step in establishing some facts about the whole structure of these systems. Triangular chiral complexes and attractors documented in our previous paper represent the initiator phase of this sequential pivotal event (Figures 1A, $1 \mathrm{~B}, 1 \mathrm{C}$, and 1D). That continues through propelled ejected crystal units from the triangular mirror core geometry, and describes trials of uniform spatial translations and interconnections in the interstitial microenvironment (Figures $1 \mathrm{E}, 1 \mathrm{~F}$, and $1 \mathrm{G})$. The driving force behind the development of these systems, ie, the seeding, evolutionary growth, and reseeding model, is a process description of how this happens. This pattern emerges in oblique space orientation, in relation to the trail trajectory of the microcrystal ejected from the core of the system, in relation to the high friction that leaves the interstice and represents the journey of the crystal molecule in an interval space of time that we have called CCET.

CCET originates from the fast pulsar-like spin rotation in the opposite orientation of the overlapping triangular mirror-crystallized cells from perturbated unstable collision molecules, such as a beam projection from the core of the hexagonal pattern. This demonstrates the electromagnetic origin of the jets, ie, the microcrystal jets come directly from a rotating core, and it is the magnetic stresses caused by the rotation that focus and accelerate the flow of the jet. Statistical analysis found that for 1077 GTCHC complexes, 915 of these have a CCET pattern, which is $85 \%$ of the sample $\left(\chi^{2}=38.63, P=0.000001\right)$ and it was negative in $15 \%$ (162 cases, Table 1 ). In this entangled environment, the states of two or more objects become so inextricably connected that none of them can be described separately. When two entangled objects are separated, as soon as the state of one is measured, the state of the other is instantly known. For this reason, we can document the crystals ejected from
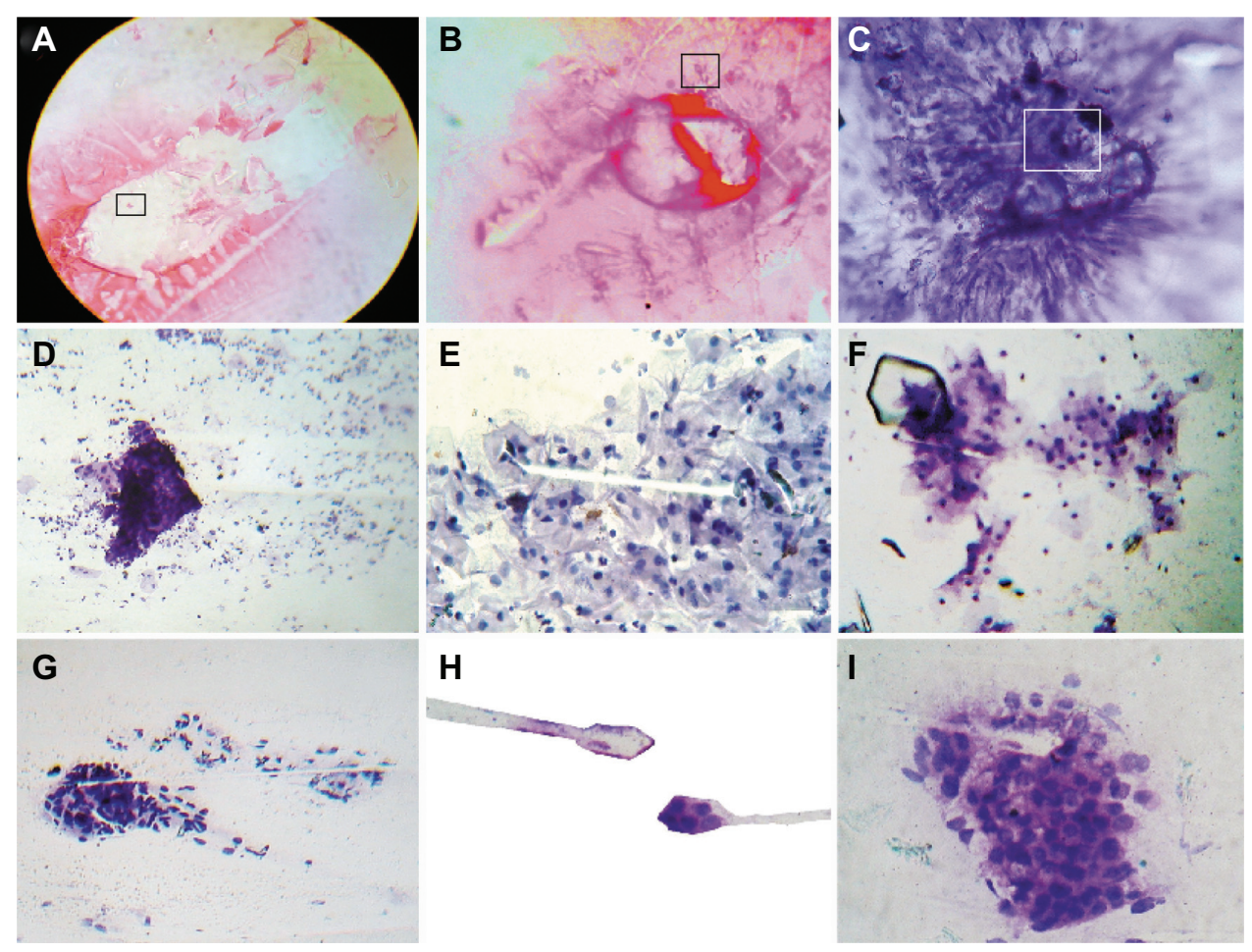

Figure I Initiator phase of geometric triangular chiral hexagonal like crystal complex assembly in malignant tumors. A and $\mathbf{B}$ show a geometric triangular chiral hexagonal-like crystal complex assembly in ascitic fluid in a case of abdominal carcinoma with Papanicolaou staining (40x); C and $\mathbf{D}$ show the triangular mirror image assembly in a case of endocervical adenocarcinoma, endocervical smear with Papanicolaou staining (40x, observe the black frame in A-C); E-G illustrate the crystal comet effect tail from the core of the hexagonal pattern in a case of cervical carcinoma in situ, vaginal smear with Papanicolaou staining (40x); $\mathbf{H}$ and I show the crystal comet effect tail with a simultaneously opposite crystal trajectory in a case of papillary thyroid carcinoma, fine needle aspiration, Papanicolaou staining (40×). 
Table I Relationship between geometric triangular chiral crystal complexes and crystal comet effect tail assembly

\begin{tabular}{lll}
\hline GTCHC & CCET-positive & CCET-negative \\
\hline 1077 & 915 & 162 \\
& $85 \%$ & $15 \%$ \\
\hline
\end{tabular}

Abbreviations: CCET, crystal comet effect tail; GTCHC, geometric triangular chiral crystal complexes.

hexagon geometry, the simultaneously opposite influx crystal arrival trajectory, and side by side as a dynamic bipolar mechanism arises (Figures $1 \mathrm{H}$ and 1I). This dual trajectory generates, in time lapse sequence, triangular chiral mirror images (Figures 2A and 2B) and state-of-the-art complexes (Figures 2C and 2D). Cancer microcrystals in this campaign leave the ensembles of interacting components. The stellar trail trajectory generates a powerful electromagnetic field alongside this crystalline line. As soon as large amounts of entangled objects form, the spinning of microcrystal particles, like magnetic material, forms spin domains that rotate in opposite directions. The alignment of those spins forms many possible spin configurations, which are the connection interface point for biological elements. It is the pivotal point situation in which several different physical and biological interactions converge. In this process of magnetism and spiral behavior, the crystal molecules vibrate and grow. By overlapping, the structures increase to generate multilayer copies of self, or form a fractal replication assembly repeating many times over time.

In the transformation phase, as the growth phase progresses, the total thickness of the multilayer microcrystal increases and starts to replicate, creating a new complex from the crystal. Following this same pattern, a geometrically regular three-dimensional structure arises due to the dense distribution associated with the electromagnetic properties of the structure. It becomes more complex, and fractal structures that represent the echo images of crystal growth vibration appear, generated by the rotation spiral of electromagnetic waves. This is the convergent and confluent phase of the system.

As more and more layers of fractal crystals are incorporated to the system, it becomes more complex until it reaches the point that the final master construction, ie, the embryoid body phenotype, is easily recognizable (Figures 2E, 2F, 2G, $2 \mathrm{H}$, and $2 \mathrm{I}$ ). We identified the embryoid body phenotype

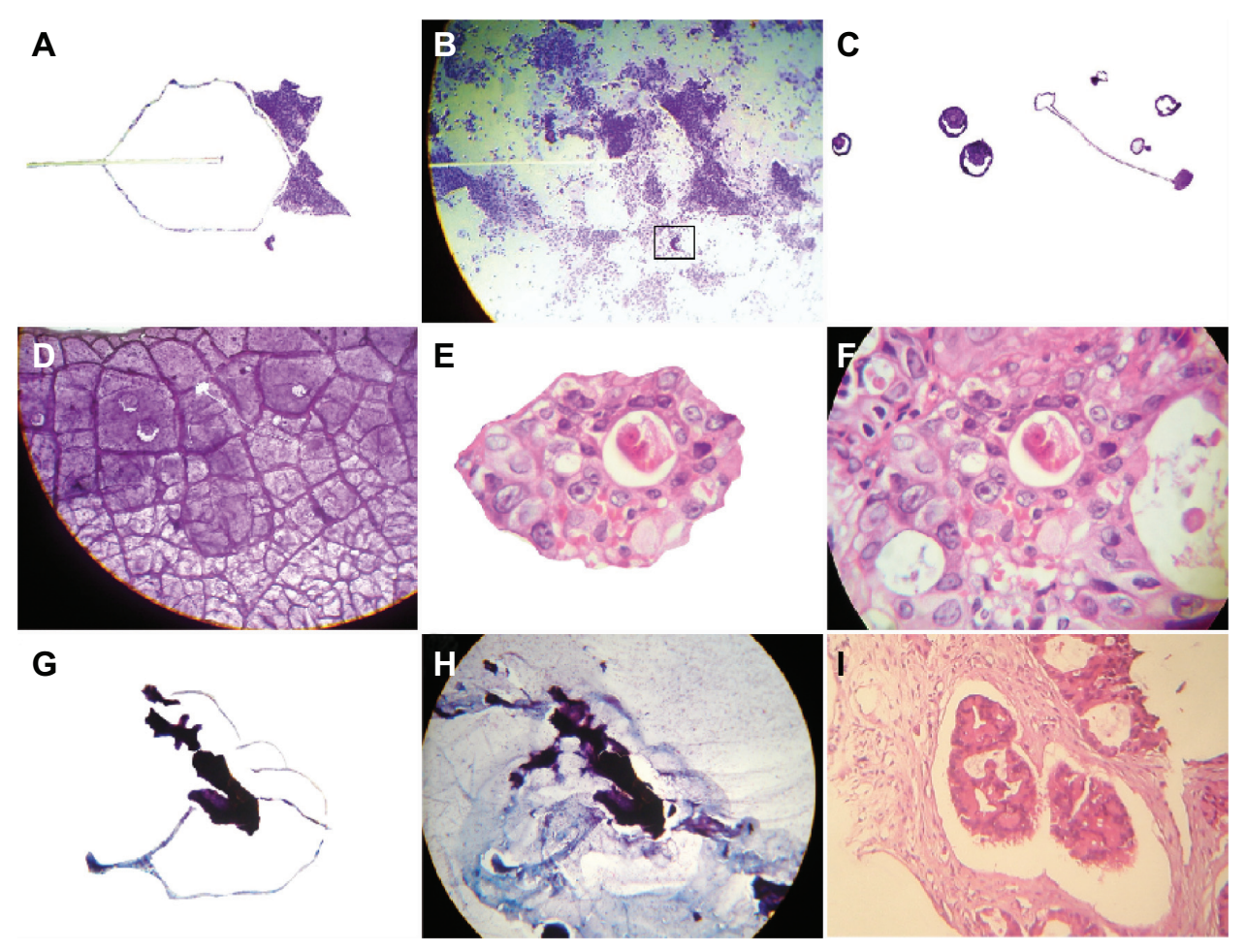

Figure 2 The crystal comet effect tail generates triangular mirror images and a state-of-the-art complex. A shows the detachment subimage of $\mathbf{B}$, which illustrates the triangular mirror image generated from the crystal comet effect tail in a case of endometrial adenocarcinoma, Papanicolaou staining (40×, observe the detail area inside the black frame); $\mathbf{C}$ shows the detachment subimage of $\mathbf{D}$, which is a state-of-the-art complex in a case of mucinous cystadenocarcinoma of the ovary, ascitic fluid, Papanicolaou staining (40x); $\mathbf{E}$ is a detachment subimage of $\mathbf{F}$, which shows embryoid body self-assembly into a geometric triangular chiral hexagonal like crystal complex in a case of colon adenocarcinoma with hematoxylin and eosin staining (20x); $\mathbf{G}$ is a detachment subimage of $\mathbf{H}$, which shows fractal embryoid bodies ejected from the core of the hexagonal geometric complex, generating buoyant vibrational echo image waves in a case of breast adenocarcinoma, fine needle aspiration, Papanicolaou staining (20x); and I shows well-defined fractal embryoid bodies in a case of breast adenocarcinoma with hematoxylin and eosin staining (20x). 
and its different developmental stages, from early precursor cleavage of chiral triangular mirror images to transitional forms and, finally, recognizing it at a microscopic level (Figures 3A and 3B). Furthermore, we were able to ascertain how embryo transformations are structured inside and in intimate relation with the geometry of GTCHC and CCET complexes (Figures 3C and 3D). We are also able to observe and track the sequential cellular development phases of adenocarcinoma, from the malignant glandular cells lining the transformed epithelium and fractal replication resonance copy images (Figures 3E, 3F, 3G, 3H, and 3I). In the process of self-assembly and fractal self-replication, the embryoid body continuously appears as a well-defined construction with spatially recognizable clear differentiation (Figures 4 and 5), and was used as scaffolding to grow cells in twodimensional and three-dimensional macroscopic arrays (Figures 6A, 6B, 6C, 6D, 6E, 6F, 6G, and 6H) to the point where we were able to isolate them (Figure 6I). Statistical analysis showed that 549 of the 915 CCET assemblages had an embryoid pattern, comprising $60 \%$ of the sample $\left(\chi^{2}=34.59, P=0.000001\right)$, and $366(40 \%)$ did not have an embryoid pattern (Table 2).

\section{Enolase immunostaining}

We observed a highly immunostain-selective distribution of the neural antibody related to clusters of template triangular chiral mirror-differentiated malignant cells organized in an embryoid body phenotype assemblage. There was positive antibody activity in relation to the chiral pattern organization of groups of cells in the context of the restricted area of the tumor. Thus, enolase can be used to track and facilitate embryoid body recognition (Figures 7A, 7B, 7C, 7D, 7E, 7F, 7G, 7H, and 7I).

Statistical analysis determined that 67 of the 90 embryoid patterns $(75 \%)$ had high immunopositivity for neuronspecific enolase $\left(\chi^{2}=17.16, P=0.000034\right)$, and was negative in 23 cases $(25 \%$, Table 3$)$.

\section{Collision event}

The natural environment in terms of atmospheric space, time, and light conditions is the best laboratory setting to carry out electromagnetic field research. In this experimental model, the collision of a strong flash from a white light against an electromagnetic field with electric conduction lines produced morphodynamic sequential collider partner images.

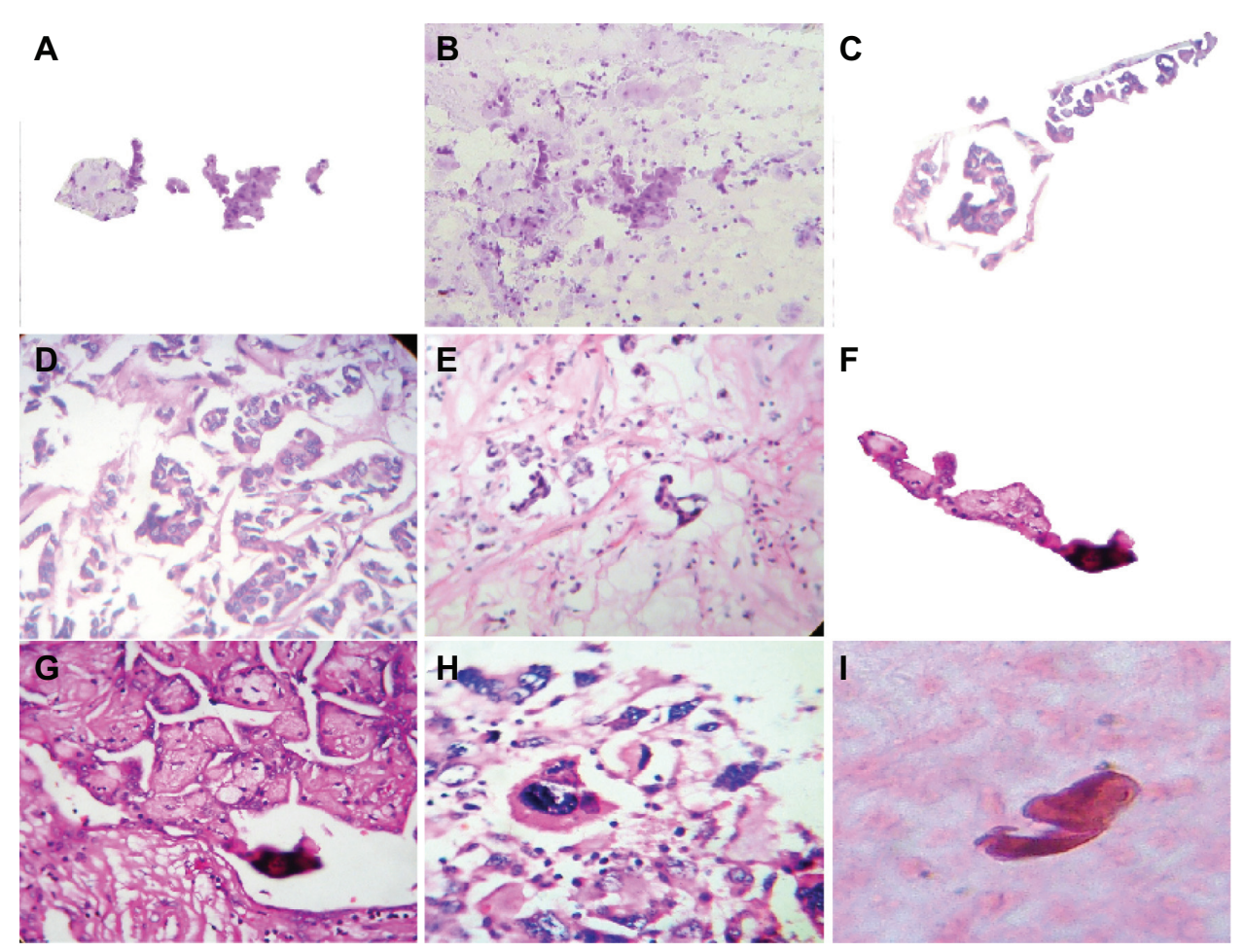

Figure 3 The crystal comet effect tail generates alignment of fractal embryoid bodies. A shows a detachment subimage of B, demonstrating fractal embryoid body alignment emerging from a hexagonal core geometric organization in a case of Grade I cervical intraepithelial neoplasia with Papanicolaou staining (I0x); C is a detachment subimage of $\mathbf{D}$ which is a micrograph displaying assembly of well-defined fractal embryoid bodies in a case of gastric adenocarcinoma with hematoxylin and eosin staining (20x); $\mathbf{E}$ is a micrograph displaying assembly of well-defined fractal embryoid bodies in a case of colon adenocarcinoma with hematoxylin and eosin staining (20x); $\mathbf{F}$ is a detachment subimage of $\mathbf{G}$, which shows a mirror image of well-defined fractal embryoid bodies in a case of renal cell carcinoma with hematoxylin and eosin staining (20x); $\mathbf{H}$ shows a fractal embryoid body in a case of undifferentiated sarcoma tumor with hematoxylin and eosin staining (20x); I shows self-assembled embryoid body with a well-defined pattern of formation in a necrotic area of leiomyosarcoma, with hematoxylin and eosin staining (20x). 

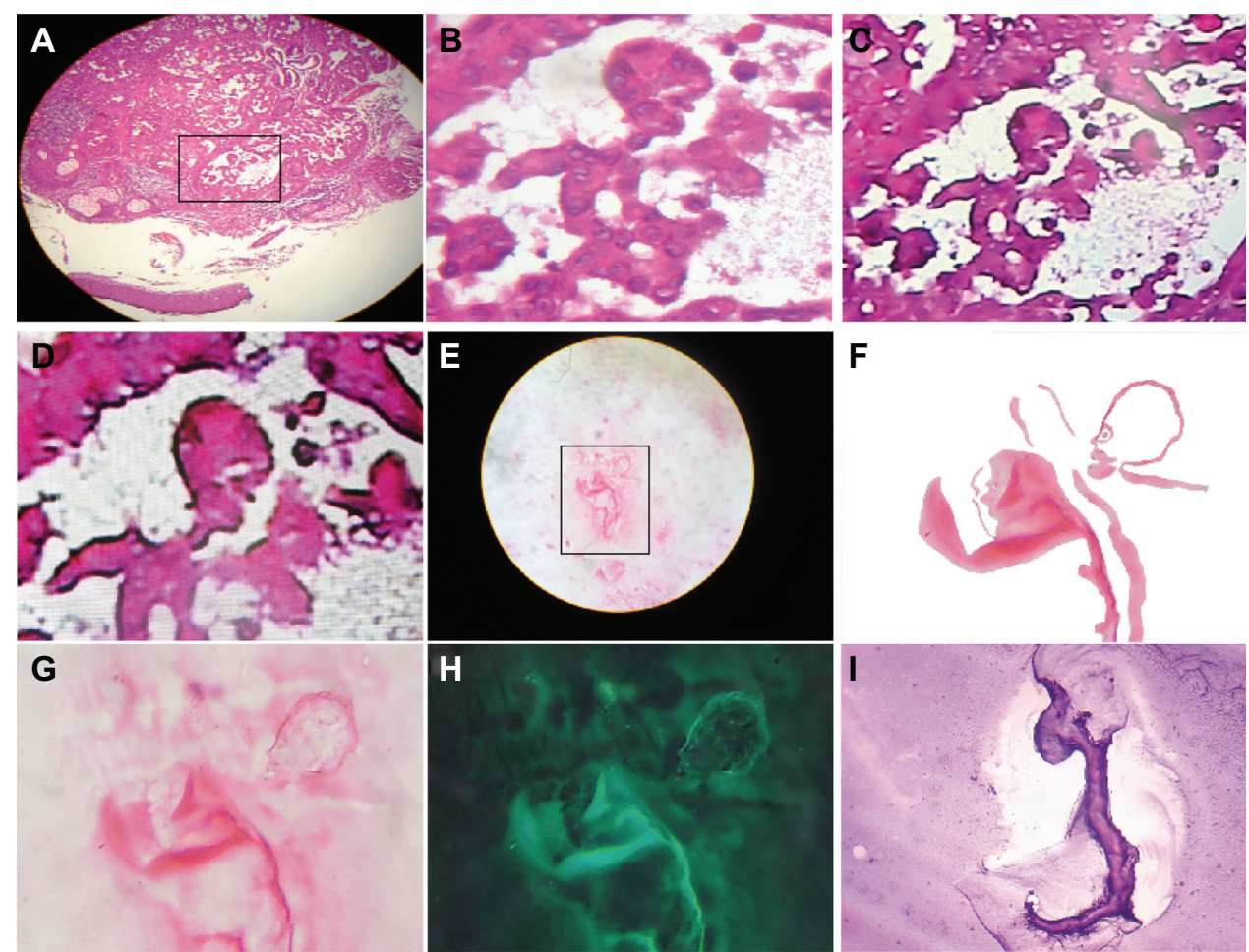

Figure 4 Well-defined self-assembled embryoid body pattern formation. A-D are closeup images of a well-defined embryoid body pattern formation in a case of squamous cell carcinoma of the skin, hematoxylin and eosin staining (20x); E-G show a well-defined, self-assembled embryoid body pattern formation in a case of peritoneal carcinoma with ascites, Papanicolaou staining (20x); $\mathbf{H}$ is a negative image of $\mathbf{G}$; I reveals embryoid body self-assembly with geometric triangular chiral hexagonal crystal as a template platform in a case of peritoneal carcinoma with ascites and Papanicolaou staining (20x).
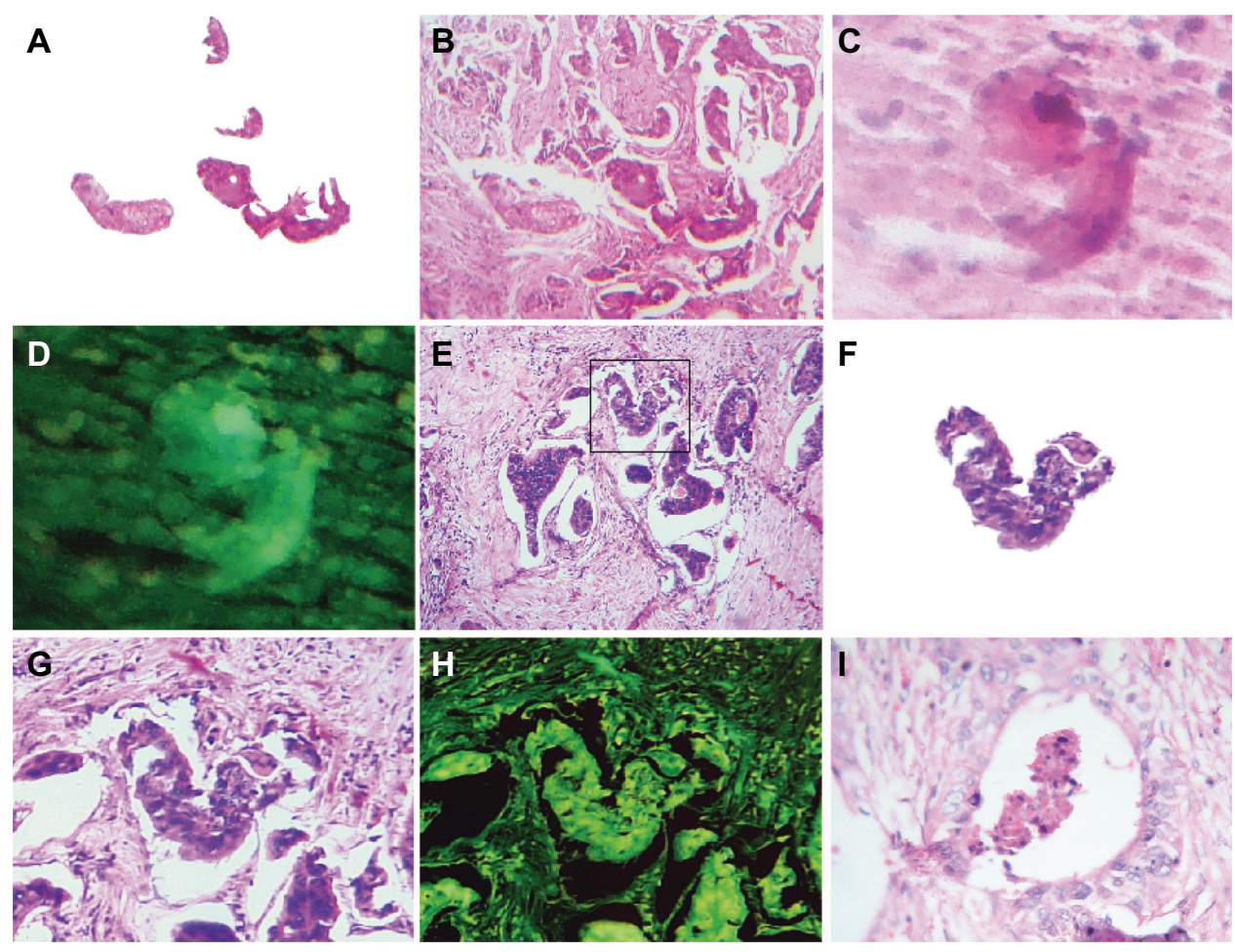

Figure 5 High-gradient self-assembled sequential embryoid body pattern formation. A is a detachment subimage of B, which shows high-gradient sequential embryoid body pattern formation from a case of breast adenocarcinoma, hematoxylin and eosin staining (20x); $\mathbf{C}$ shows a sequential embryoid body pattern with well-defined formation from a necrotic area in an undifferentiated sarcoma tissue, hematoxylin and eosin staining (20x); $\mathbf{D}$ is a negative image of C; E-G are closeup views of a well-defined embryoid body pattern formation in a case of breast adenocarcinoma, hematoxylin and eosin staining (20x); $\mathbf{H}$ is a negative image of $\mathbf{G}$; I shows a well-defined pattern of embryoid body formation in a case of prostate adenocarcinoma, hematoxylin and eosin staining (20x). 

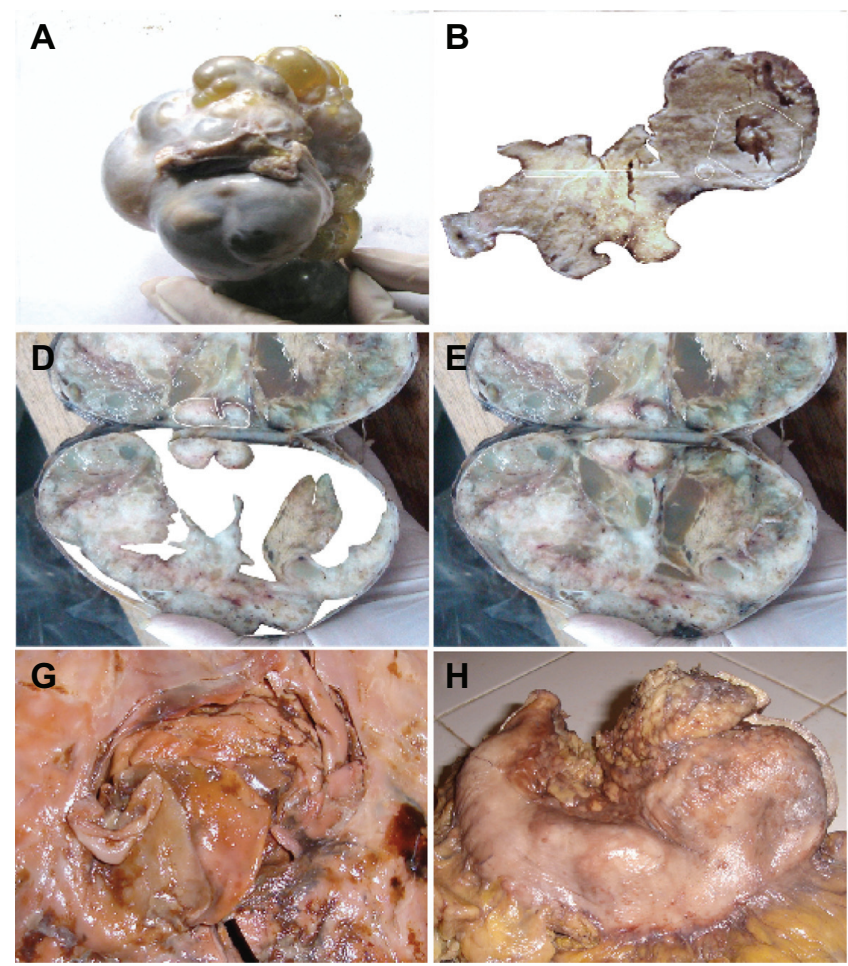
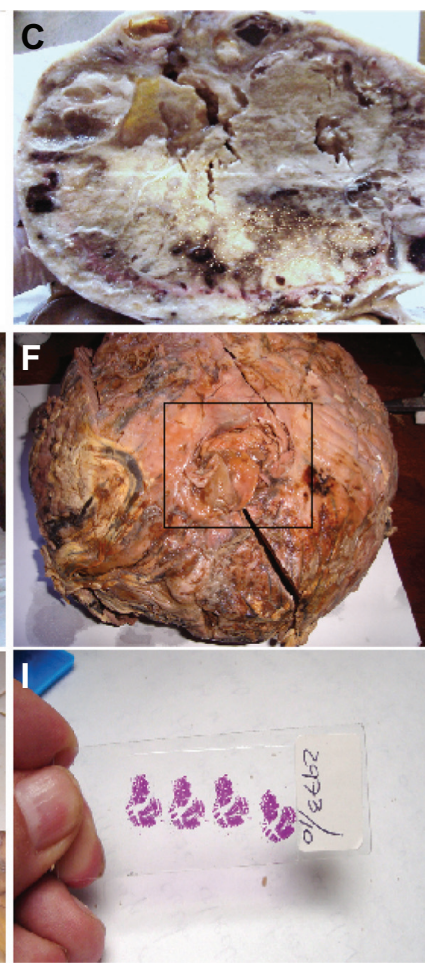

Figure 6 Macroscopic representation of an embryoid body. A is a macroscopic representation of a fractal embryoid body in a case of malignant ovarian tumor; B is a detachment subimage of $\mathbf{C}$, a macroscopic representation of an embryoid body revealing a hexagonal geometric pattern and a crystal comet tail from a case of retroperitoneal malignant tumor; $\mathbf{D}$ is a detachment subimage of $\mathbf{E}$, which is a macroscopic embryoid body representation from a case of renal cell carcinoma; $\mathbf{F}$ and $\mathbf{G}$ are macroscopic representations of fractal embryoid body structures from a case of leiomyosarcoma; $\mathbf{H}$ is a macroscopic representation of an embryoid body from a case of diffuse gastric cancer; I shows is an embryoid body from a case of squamous cell carcinoma of the skin, hematoxylin and eosin staining.

With the introduction of variants in space (in which the angle of incidence of the flash discharge was modified) or time (period of the day in which the experiment was carried out), the resulting geometric images varied at the region of interaction. Accordingly, we modified the hour when the experiments were conducted. Our previous experiments were carried out in a completely dark environment, but in this case, the experiment was performed at dawn. We were able to reproduce a perfect comet effect whereby ejected particles of a light wave followed a straight line from a hexagonal core geometry close-up of this luminous energy trajectory, which was obtained through a chain of hexagonal light cluster images that allowed us to observe its structure (Figures 8A and $8 \mathrm{~B}$ ). In space-time interval modes, this chain was separated and it was possible to identify spin domains separated

Table 2 Relationship between crystal comet effect tail assembly and embryoid body pattern

\begin{tabular}{lll}
\hline CCET & $\begin{array}{l}\text { Embryoid pattern } \\
\text { positive }\end{array}$ & $\begin{array}{l}\text { Embryoid } \\
\text { pattern negative }\end{array}$ \\
\hline 915 & 549 & 366 \\
& $60 \%$ & $40 \%$
\end{tabular}

Abbreviation: CCET, crystal comet effect tail. from the principal base chain at the interior of the hexagonal cluster generated by fast rotation of the light particles. In the opposite orientation triangular mirror images, from the core of this energy, a jet flow stripe line emerged from the entangled light particles, making it possible to identify chains of hexagonal patterns that resembled the hexagonal chain of the original mold, which were identical to the ones observed in tumors (Figures 8C, 8D, 8E, and 8F). In the final sequence event, the overlapping photons, interacting with straight lines of electromagnetic waves, reproduced light optical fractal resonances that were organized in identical plasma magnetic circuit patterns, and identified in embryoid bodies (Figure 8G, 8H, and 8I). Surprisingly, when comparing the essential assemblage patterns generated through electrooptical collision, we found similarities with cancer tissue at the macroscopic and microscopic levels. The concordance between the images from the experimental model and the tumor are conclusive.

\section{Discussion}

It is very difficult to explore chaotic systems on the basis of purely theoretical or mathematical models or from computational tools because it is not possible to observe the 


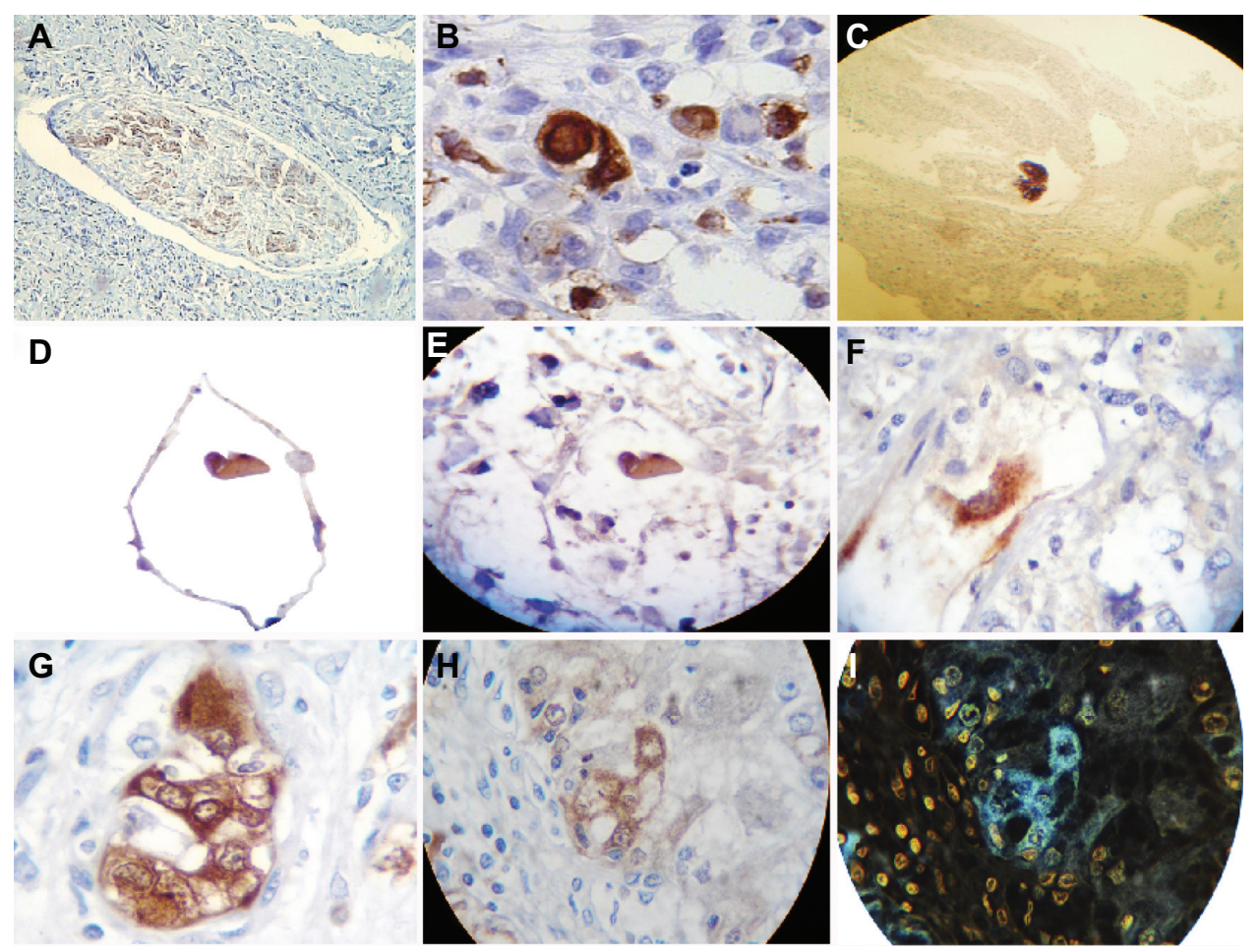

Figure 7 Neuron-specific enolase immunostaining analysis. A shows neuron-specific enolase immunostaining of control tissue from a peripheral nerve (I0x); B reveals highly selective enolase immunopositivity in aligned fractal embryoid body structures from a case of esophageal squamous cell carcinoma (40×); $\mathbf{C}$ is a panoramic view revealing highly selective enolase immunopositivity in a self-assembled fractal embryoid body structure aligned into a hexagonal geometric pattern from a case of skin squamous cell carcinoma (I0X); D is a detachment subimage of $\mathbf{E}$, which shows highly selective enolase immunopositivity in a self-assembled embryoid body structure inside a hexagonal geometric pattern in a case of lung carcinoma (I0X); $\mathbf{F}$ is a micrograph demonstrating highly selective enolase immunopositivity in a well-defined self-assembled embryoid body pattern in a case of undifferentiated lung cancer (20x); G is a micrograph revealing high enolase immunopositivity in a well-defined self-assembled embryoid body pattern from a case of prostate carcinoma (40x); $\mathbf{H}$ is a micrograph revealing enolase immunopositivity in a well-defined, self-assembled embryoid body pattern in a case of squamous cell carcinoma of the skin $(I 0 \times)$; and $\mathbf{I}$ is a negative image of $\mathbf{H}$.

multitude of interrelationships that exist in a living cancer system. In concordance with our images in the final step of the natural history of cancer, a complex system that appears initially to be "chaotic" could find order eventually. This shows how a large number of independent unpredictable events can combine together and reassemble in order to yield predictable and orderly behavior. From a panoramic multifocal vision, we decoded the encrypted structural puzzle hidden in these geometric complexes that evolve over time in four sequential steps (Figure 9).

\section{Initiator phase}

This phase begins from the moment at which thousands of malignant cellular membranes embark on a gradually increasing collision process as these cells multiply in disorder.

Table 3 Embryoid body selective distribution of neuron-specific enolase antibody immunopositivity

\begin{tabular}{lll}
\hline Embryoid body & Enolase-positive & Enolase-negative \\
\hline 90 & 67 & 23 \\
& $75 \%$ & $25 \%$ \\
\hline
\end{tabular}

Molecular biologic crystals, represented by triangular chiral hexagons derived from collision attraction events against collagen type I fibrils, emerge at microscopic and macroscopic levels. This phase is full of geometric complexes. The essential basis of this geometry is the triangulation and generation of mirror images that conclude in hexagons. These hexagons are not simple six-sided structures, but are in a complete state, net balanced, and structured in the base of three triangular units, with their respective mirror images in a state of molecular order. GTCHC is a mechanism of structural initiator assembly, a template organizer at each side of hypertrophy helicoid fibers that represent flow energy during cooperative hierarchical chiral electromagnetic interactions in pathological tissues. Through repeat assemblages of triangles inside triangles, the system makes error corrections through selfassembled triangular mirror patterns which introduce fractal order, eliminate errors, and purge the system. This is not a new concept, and is known as fractal imaging, and also as the Sierpinski triangle, ie, a type of crystal or structure that regularly repeats itself, and a pattern of triangles that looks the same under any view. Molecules direct the process of 

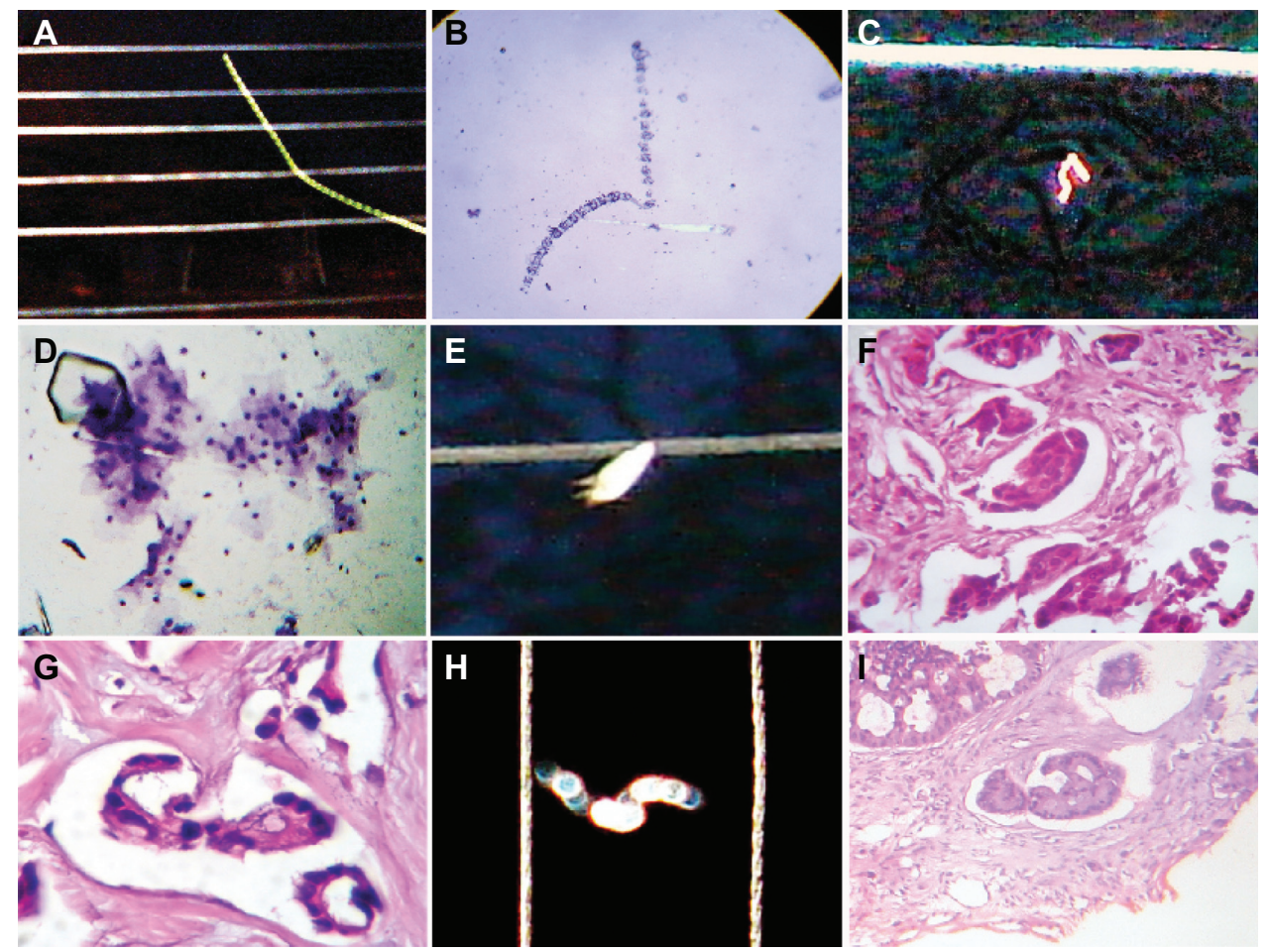

Figure 8 Comparative image analysis of reproducible and predictable template platforms generated by the electro-optical collision model. A shows a reproducible and predictable template platform of a comet tail, generated by the electro-optical collision model; B is a comparative image analysis of ascitic fluid from a case of ovarian carcinoma, with Papanicolaou staining (I0X); C shows a reproducible and predictable template platform for geometric triangular chiral hexagonal-like crystal complexes generated by the electro-optical collision model; $\mathbf{D}$ is a comparative image analysis of a vaginal smear for a case of cervical carcinoma in situ, Papanicolaou staining (20x); $\mathbf{E}$ shows a reproducible and predictable template platform plasma magnetic circuit pattern generated by electro-optical collision; $\mathbf{F}$ comparative image analysis of a case of breast carcinoma, hematoxylin and eosin staining (20x); G shows well-defined pattern formation of an embryoid body in a case of colon adenocarcinoma, hematoxylin and eosin staining $(20 \times)$, with comparative image analysis and a reproducible and predictable template platform plasma magnetic circuit pattern generated by electro-optical collision in $\mathbf{H}$, which again shows a reproducible and predictable template platform plasma magnetic circuit pattern generated by electro-optical collision; I shows a welldefined embryoid body pattern formation in a case of colon adenocarcinoma, hematoxylin and eosin staining (20x), and comparative image analysis with a reproducible and predictable template platform plasma magnetic circuit pattern generated by electro-optical collision in $\mathbf{H}$.

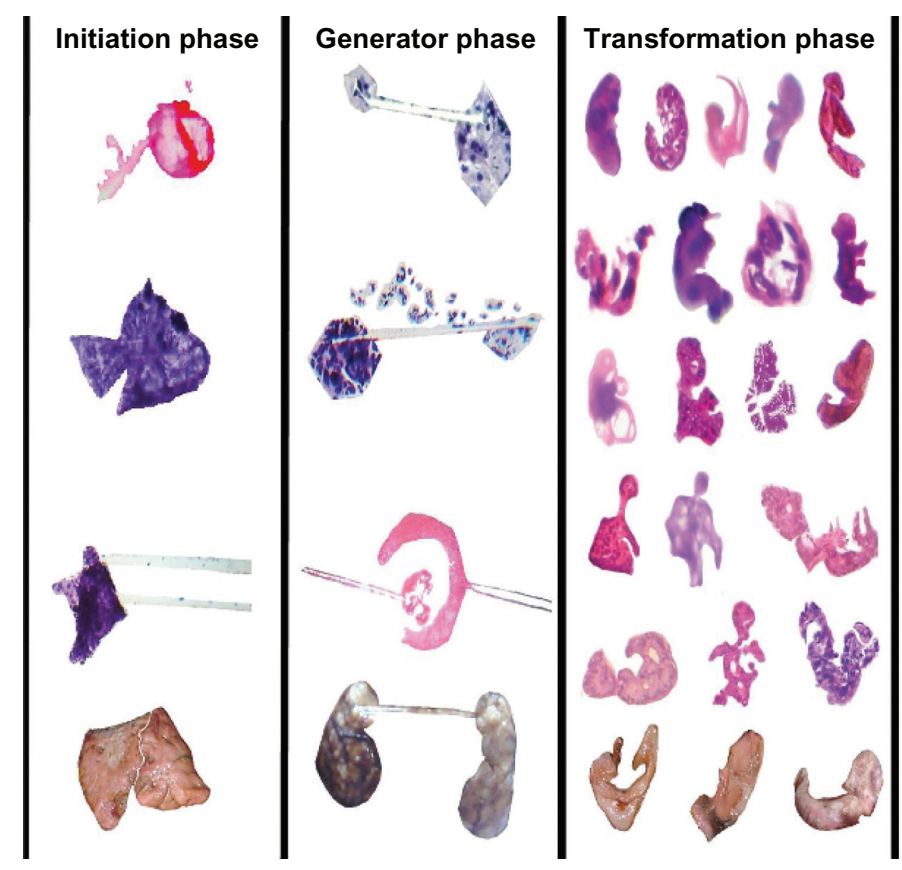

Diferentiation phase
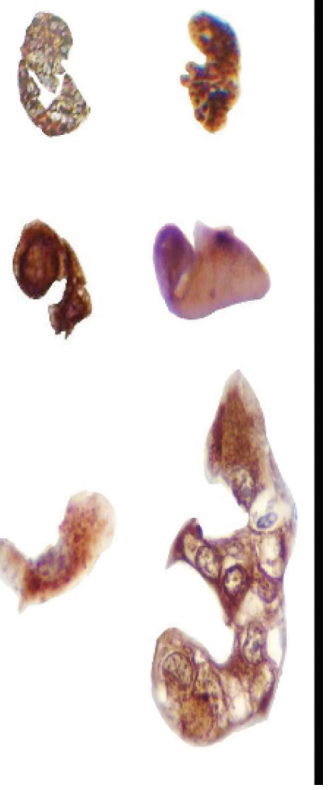

Figure 9 Sequential development of embryoid body pattern. 
self-assembly and are successful in reducing assembly errors to insignificant levels. Winfree et al reported that DNA "tiles" can be programmed to assemble themselves into a crystal bearing a pattern of progressively smaller "triangles within triangles". ${ }^{10}$

\section{Generator phase}

Electromagnetic forces are dominant in forming biologic molecules. Colliding pairs of spirals that are oriented in opposite directions generate powerful electromagnetic fields in the collision interaction region, ejecting microcrystal particles with wave light electromagnetic trails split into two components that follow opposite directions in a helicoid flow pattern with polarization and mirror imaging. Fractioned crystals ejected from the core of the system rotate on their axes, generating a magnetic field as they do so. The projective line, in essence, is the visual expression of the trajectory of ejected microcrystals in the environment, and is formed by integrating the modes of perfectly aligned sequential triangular-hexagonal biologic crystal particles with high chiral order.

\section{Fractal transformation}

Beginning with the alignment of spin domains under the magnetic field, in which all domains point in one direction in relation to the initial collision event, fractals represent the echo image of crystal growth vibration generated by the spiral rotation of electromagnetic waves, and is the sum of the total fractal resonances, which is the convergent and confluent phase of the system. Complex molecular matter is then organized into the fractal form, which is the ultimate expression of interaction of all forces, and involves the distribution of electromagnetically charged biologic molecules and mass aggregations (gravitational field) that result in a final master construction. ${ }^{11}$ Clearly, exogenous forces, ionic molecules, nutrient content, flow of nutrients, central/distal, proximal/distal, even left/right molecules, provide sufficient forces to cause cancer cells to differentiate. Differentiation accelerates as the complexity grows.

\section{Differentiation phase}

We wondered about the significance of the alignment we repeatedly observed in our images. Previous studies have indicated that cell alignment is required for differentiation ${ }^{12}$ in an evolutionary state of morphogenesis in different species. Many observations enable us to affirm that triangular chiral mirror-aligned structural images represent the template base axial polarization related to the chiral disposition of some types of differentiated malignant cells that become enolase-positive when their embryoid bodies are assembled. Moreover, the ones that do not show template base polarization are enolase-negative. We observed a highly immunostainselective distribution of the neural antibody related to clusters of template triangular chiral mirror differentiated malignant cells organized in an embryoid body phenotype assemblage. Immunostaining consistently showed individual differentiated cells in the context of the whole tumor. Here we detected antibody activity in relation to the chiral pattern organization of groups of cells in the context of the restricted area of the tumor. Polarity reveals intrinsic chirality. ${ }^{13}$

Embryoid bodies represent the final expression of this convergent flow of information through triangular chiral crystal molecules (ie, GTCHC) and assembly in a light optic polarized electromagnetic corridor (ie, CCET). It is evident in all of our images that these embryoid bodies are inside or in intimate contact with geometric complexes. Scientists at the University of Chicago have successfully used geometrically patterned surfaces to influence the development of stem cells. ${ }^{14}$ Recent studies have demonstrated how light can make the cells move in directional aligned patterns. ${ }^{15}$ Additionally, in a recent study, Raman spectroscopy, which measures the scattering pattern of light in a system or compound, showed that differentiation between a benign breast lesion and a malignant one can be done based on chemical or crystal composition and tissue morphology, as well as the respective light scattering spectrum patterns. ${ }^{16}$ Thus, the alignment characteristics of cancer tissues documented extensively in this article is probably due to light electromagnetic regulation.

Ultraviolet light helps to form the building blocks of life by adding ultraviolet light to a model prebiotic reaction, and researchers from the Georgia Institute of Technology and the University of Roma have discovered a route by which the missing guanine could have been formed. ${ }^{17}$ They have also found that the RNA bases may be easier to form than previously thought, suggesting that starting life might not have been difficult after all. It is evident that light creates the structural platform for the fundamental molecules necessary to generate life.

It is well known that serum from individuals with colorectal, gastric, pancreatic, lung, or breast carcinoma, as well as individuals with medullary thyroid carcinoma contains higher levels of carcinoembryonic antigen than serum from individuals without cancer. ${ }^{18}$ In addition, alpha-fetoprotein is seen in hepatocellular carcinoma, germ cell tumors, metastatic liver cancer, and several other primary tumors. ${ }^{19}$ 
This is normally produced during fetal development, and production stops before birth. Therefore, it is not usually present in the blood of healthy adults. It is probable that the origin of such proteins is elaborated by cancer stem cells, a very challenging population of cells to identify. For researchers, the identification, characterization, and isolation of embryoid bodies in tumor pathology is probably intimately linked with cancer stem cells. In concordance with our observations, at the center of every voluminous tumor, if malignant groups of enolase-immunopositive differentiated cells form conglomerates that adopt visible microscopic and macroscopic embryoid patterns, it is possible that cancer cells could contain the genetic information for primitive embryogenic cell reassembly, which indirectly demonstrates the existence of molecular ancestral memory that permits such regression by transmitting heritable information to where most of our primitive genes are reactivated. This organization is related to how the cells take up a fractal gravitational position in space, and how for the first time the proteins take up determinantspecific localization inside limited space in order to generate life, which is possible only by collective cell behavior in which each part contributes to the formation of a pattern in an overlapping and coalescent event. Cancer can give us important clues about the nature and history of life itself.

We want to analyze the significance of these bodies from two opposite viewpoints. First, embryoid bodies are a seeder and feeder of a proliferative tumor. In this way, we observe how these structures are located in cavities surrounded by a protective watery barrier, where it could remain probably untouched by the immunological system, chemotherapy, or radiotherapy, and could cause relapse of the tumor.

The other viewpoint is that if nature is organized in fractal geometric patterns, ${ }^{20,21}$ why are human cells and specialized tissues exempt from this law? How does the natural history of cancer permit this fractal organization? ${ }^{22-26}$ Our findings suggest that cancer is a system that aims for the destruction of the existing order, creating self-arranged cellular differentiation. During this process, the architecture collapses and fragmental components emerge, revealing their hidden interior and how each molecule is reassembled into the original mold, using one common connection, ie, fractal self-similarity, which guides the system from the beginning. GTCHC complexes generate ejected CCTE and strange helicity states arising in the form of spin domain interactions. As the crystal growth vibration stage progresses, biofractal echo images converge into a master built construction of embryoid bodies with enolase-selective immunopositivity in relation to clusters of organized triangular chiral cells. Using our electro-optic collision model, we were able to predict and replicate all the characteristics of the complex geometry that connects a physical phenomenon with the signal patterns that chaos biology generates. Intrinsically, fractal geometry makes the spatial correction errors of this chaotic system in a way that permits new structures to emerge. As a result, an orderly selfassembly of embryoid bodies with neural differentiation as the final stage of cancer development becomes a predictable process. Recursive ontogeny recapitulates an earlier stage of development, but in a different exogenous environment in which the macrostructure is a fractal dimension of infinite replication series of self-similarity microstructures.

We hope that further investigation of these structures will lead not only to a new way of thinking about physics and biology, but also to a rewarding area of cancer research to develop new strategies and therapeutic tools to fight this disease.

\section{Acknowledgments}

The authors thank Dr Jesus Rosado and Dr Jorge Ivan Cruz, executive directors of the Hospital Department of Villavicencio, and Hospital Department of Granada, respectively, for their financial support of this research and help with logistics.

\section{Disclosure}

The authors report no conflicts of interest in this work.

\section{References}

1. Rose GG. Crystals and particles produced in tissue culture: I. Introduction. Cancer Res. 1963;23:279-284.

2. Rose GG. Biologic crystals and particles produced in tissue culture: II. Enzymatic responses and environmental transformations. Cancer Res. 1964;24:1159-1193.

3. Díaz JA, Jaramillo NA, Murillo MF. Geometric triangular chiral hexagon crystal-like complexes organization in pathological tissues biological collision order. 2007;2:e1282.

4. Díaz JA, Jaramillo NA, Murillo MF. Framework of collagen type I vasoactive vessels structuring invariant geometric attractor in cancer tissues: Insight into biological magnetic fields. 2009;4:e4506.

5. Patel AB, Gibson WT, Gibson MC, Nagpal R. Modeling and inferring cleavage patterns in proliferating epithelia. PLoS Comput Biol. 2009;5: e1000412.

6. Cairns-Smith AG. Genetic takeover and the mineral origins of life. Genet Res. 1988;51:167-168.

7. Toor N, Keating KS, Taylor SD, Pyle AM. Crystal structure of a self-spliced Group II intron. Science. 2008;320:77-82.

8. Soler-Federsppiel BS, Cras P, Gheuens J, Andries D, Lowenthal A. Human gamma, gamma-enolase: Two-site immunoradiometric assay with a single monoclonal antibody. J Neurochem. 1987;48:22-28.

9. Bancroft JD, Stevens A. Theory and Practice of Histological Techniques. 4th ed. New York: Churchill Livingstone; 1996.

10. Rothemund PWK, Papadakis N, Winfree E. Algorithmic self-assembly of DNA Sierpinski triangles. PLoS Biol. 2004;2:e424.

11. Tarasov VE. Gravitational field of fractal distribution of particles. Celestial Mechanics and Dynamical Astronomy. 2006;94:1-15. 
12. Kim SK, Kaiser D. Cell alignment required in differentiation of Myxococcus xanthus. Science. 1990;249:926-928.

13. Xu J, Van Keymeulen A, Wakida NM, Carlton P, Berns MW, Bourne HR. Polarity reveals intrinsic cell chirality. Proc Natl Acad Sci U S A. 2007;104:9296-9300.

14. Kilian KA, Bugarija B, Bruce T, Mrksich L. Geometric cues for directing the differentiation of mesenchymal stem cells. Proc Natl Acad Sci US A. 2010;107:4872-4877.

15. Dogariu A, Sugaya K. Scientists control living cells with light; advances could enhance stem cells' power. Available at: http://www.sciencedaily. com/releases/2009/08/090811161347. Accessed November 29, 2009.

16. Haka AS, Volynskaya Z, Gardecki JA, et al. In vivo margin assessment during partial mastectomy breast. Cancer Res. 2006;66:3317-3322.

17. Barks HL, Buckley R, Grieves GA, Di Mauro E, Hud NV, Orlando TM. Guanine, adenine, and hypoxanthine production in UV-irradiated formamide solutions: Relaxation of the requirements for prebiotic purine nucleobase formation. Chem Bio Chem. 2010;11:1240-1243.

18. Hammarstrom $\mathrm{S}$. The carcinoembryonic antigen (CEA) family: Structures, suggested functions and expression in normal and malignant tissues. Semin Cancer Biol. 1999;9:67-81.
19. Sizaret P, Martel N, Tuyns A, Reynaud S. Mean alpha-fetoprotein values of 1,333 males over 15 years by age groups. Digestion. 1977;15: $97-103$.

20. Mandelbrot B. The fractal geometry of nature. San Francisco, CA: WH Freeman; 1982.

21. West GB, Brown JH, Enquist BJ. The fourth dimension of life: Fractal geometry and allometric scaling of organisms. Science. 1999;284: 1677-1679.

22. Less JR, Posner MC, Skalak TC, Wolmark N, Jain RK. Geometric resistance and microvascular network architecture of human colorectal carcinoma. Microcirculation. 1997;4:25-33.

23. Baish JW, Rakesh KJ. Fractals and cancer. Cancer Res. 2000;60: 3683-3688.

24. Sedivy R. Fractal tumors: Their real and virtual images. Wien Klin Wochenschr. 1996;108:547-551. German.

25. Sedivy R, Windischberger C. Fractal analysis of a breast carcinoma: Presentation of a modern morphometric method. Wien Med. Wochenschr. 1998;148:335-337. German.

26. Smolle J. Fractal tumor stromal border in a nonequilibrium growth model. Anal Quant Cytol Histol. 1998;20:7-13.
Cancer Management and Research

\section{Publish your work in this journal}

Cancer Management and Research is an international, peer-reviewed open access journal focusing on cancer research and the optimal use of preventative and integrated treatment interventions to achieve improved outcomes, enhanced survival and quality of life for the cancer patient The journal welcomes original research, clinical \& epidemiological

\section{Dovepress}

studies, reviews \& evaluations, guidelines, expert opinion \& commentary, case reports \& extended reports. The manuscript management system is completely online and includes a very quick and fair peerreview system, which is all easy to use. Visit http://www.dovepress.com/ testimonials.php to read real quotes from published authors. 